OPEN ACCESS

Edited by:

Sandra Torriani,

Università degli Studi di Verona, Italy

Reviewed by:

Matthias Sipiczki,

University of Debrecen, Hungary

Patrizia Romano,

University of Basilicata, Italy

${ }^{*}$ Correspondence:

Albert Mas

albert.mas@urv.cat

Specialty section:

This article was submitted to

Food Microbiology,

a section of the journal

Frontiers in Microbiology

Received: 02 January 2016 Accepted: 03 March 2016

Published: 16 March 2016

Citation:

Lleixà J, Martín V, Portillo MC, Carrau

F, Beltran $G$ and Mas A (2016)

Comparison of Fermentation and

Wines Produced by Inoculation of

Hanseniaspora vineae and

Saccharomyces cerevisiae.

Front. Microbiol. 7:338.

doi: $10.3389 /$ fmicb.2016.00338

\section{Comparison of Fermentation and Wines Produced by Inoculation of Hanseniaspora vineae and Saccharomyces cerevisiae}

\author{
Jessica Lleixà ${ }^{1}$, Valentina Martín ${ }^{2}$, María del C. Portillo ${ }^{1}$, Francisco Carrau ${ }^{2}$, \\ Gemma Beltran ${ }^{1}$ and Albert Mas ${ }^{1 *}$ \\ ${ }^{1}$ Departament Bioquímica i Biotecnologia, Facultat d'Enologia, Universitat Rovira i Virgili, Tarragona, Spain, ${ }^{2}$ Sección \\ Enología, Food Science and Technology Department, Facultad de Química, Universidad de la República, Montevideo, \\ Uruguay
}

Interest in the use of non-Saccharomyces yeasts in winemaking has been increasing due to their positive contributions to wine quality. The non-Saccharomyces yeast Hanseniaspora vineae is an apiculate yeast that has been associated with the production of wine with good aromatic properties. However, little is known about the fermentation dynamics of $H$. vineae in natural must and its interaction with autochthonous yeasts. In the present study, we performed semi industrial fermentations of Macabeo and Merlot musts inoculated with either $H$. vineae or S. cerevisiae. The yeast population dynamics were monitored by plate culturing, PCR-DGGE and massive sequencing techniques. The results obtained with these techniques show that $H$. vineae was able dominate the autochthonous microbiota in Macabeo must but not in Merlot must, which exhibited a larger, more diverse yeast population. The presence of $H$. vineae throughout most of the Macabeo fermentation resulted in more fruity and flowery wine, as indicated by the chemical analysis of the final wines, which demonstrated a strong presence of phenyl ethyl acetate at concentrations higher than the threshold of perception and approximately 50 times more than that produced in wines fermented with S. cerevisiae. This compound is associated with fruity, floral and honey aromas.

Keywords: non-Saccharomyces, Hanseniaspora, alcoholic fermentation, PCR-DGGE, massive sequencing

\section{INTRODUCTION}

Wine is the result of the alcoholic fermentation of grape must. Alcoholic fermentation is driven by yeasts, and it consists of the transformation of sugars present in the must, glucose and fructose, into ethanol and carbon dioxide. The yeast species Saccharomyces cerevisiae is considered to be primarily responsible for this process. S. cerevisiae is known for only metabolizing sugars via the fermentative pathway when the sugar concentration is high, even in the presence of oxygen. This phenomenon is known as the Cabtree effect (Cabtree, 1929).

Winemaking is currently changing because of an emerging interest in the use of non-Saccharomyces yeasts during alcoholic fermentation to increase wine complexity and differentiation. Non-Saccharomyces yeasts are commonly found on the grape surfaces, and these 
yeasts have been associated with spontaneous and unpredictable fermentation, which can result in arrested or sluggish fermentation and wine spoilage. Nevertheless, several recent studies have shown that these yeasts positively affect wine fermentation and the final wine. The positive role of nonSaccharomyces ranges from a better fermentation performance to improve wine quality and complexity (Fleet, 2008; Jolly et al., 2014).

Non-Saccharomyces yeasts can contribute to the sensorial profile of wine as a result of the production of various metabolites and the activity of certain enzymes that interact with the precursors of aromatic compounds, such as $\beta$-glucosidases, which are present in many non-Saccharomyces yeast but not in $S$. cerevisiae. $\beta$-glucosidases hydrolyze aromatic glycosylated precursors into free volatile compounds to improve the final wine flavor (Swangkeaw et al., 2011; Jolly et al., 2014). Many other enzymes of technological relevance are also secreted by non-Saccharomyces yeasts, such as pectinases. Enzymes with proteolytic activity are of key interest in enological fields because they facilitate the clarification process in wine and improve protein stability (Strauss et al., 2001; Maturano et al., 2012).

These yeasts have garnered interest in winemaking due to their beneficial effects and because consumers are demanding new wine styles. Many commercial yeast companies have also begun to promote mixed and sequential wine fermentations in order to satisfy consumer and producer demands. Therefore, companies have begun to thoroughly study and commercialize non-Saccharomyces strains, like Torulaspora delbrueckii or Metschnikowia pulcherrima (Jolly et al., 2014). Moreover, some of the yeast species that are being evaluated belong to Hanseniaspora spp., the main non-Saccharomyces yeasts in grape must that are considered apiculate yeasts due their cell morphology. Specifically, the yeast Hanseniaspora vineae (anamorph Kloeckera africana) of this genus has been of great interest because it produces several key aromatic compounds (Viana et al., 2011; Medina et al., 2013).

The strain of $H$. vineae used in this study was isolated from Uruguayan vineyards and selected due to its positive effect on wine fermentation and good contribution to the aroma profile of the final wine. $H$. vineae has been demonstrated to increase fruity aromas and produce a high amount of acetate esters, such as 2-phenylethyl acetate and ethyl acetate, in wines elaborated by sequential fermentation with S. cerevisiae (Viana et al., 2011; Medina et al., 2013).

In summary, the use of non-Saccharomyces yeasts to produce new wine styles has been increasing due to the different aromatic profiles obtained. The aim of this work was to compare the fermentation dynamics of $H$. vineae and $S$. cerevisiae and the different obtained wines after the inoculation of these two species. To this end, we used natural must from two grape varieties, Macabeo and Merlot, inoculated either with $H$. vineae or $S$. cerevisiae fermented in semi-industrial conditions. The yeast population dynamics were monitored by plate culturing, PCRDGGE and $18 \mathrm{~S}$ rRNA gene massive sequencing techniques. To confirm the differences between the two species, the final wines underwent a sensory evaluation, and the aromatic profile was determined.

\section{MATERIALS AND METHODS}

\section{Yeast Strains}

The commercial wine yeast strain used in this study was Saccharomyces cerevisiae QA23 (Lallemand ${ }^{\circledR}$ ). The apiculate yeast strain used in this work, $H$. vineae T02/5AF, was isolated from Uruguayan vineyards. Strain QA23 of S. cerevisiae was obtained in active dry yeast (ADY) form and rehydrated according to the manufacturer's instructions (Lallemand ${ }^{\circledR}$ ). The $H$. vineae strain T02/5AF was obtained in fresh paste form and rehydrated in the same manner as QA23 using warm water. The inoculation was in both cases $2 \times 10^{6}$ cells $/ \mathrm{ml}$ of must.

\section{Fermentation Conditions}

The Macabeo and Merlot grape varieties were fermented at the experimental cellar of the Faculty of Enology (Mas dels Frares, Tarragona Spain). The Macabeo musts were fermented in triplicate in $100 \mathrm{l}$ tanks at $18^{\circ} \mathrm{C}$, and $6 \mathrm{~kg}$ of Merlot grapes were fermented in 81 submerged cap fermentation tanks at $26^{\circ} \mathrm{C}$. The Macabeo must was submitted to a vacuum filtration process, whereas the Merlot grapes were selectively handpicked in the vineyard.

Fermentation activity was followed by daily density monitoring using a portable densimeter (Mettler Toledo). Samples were taken once a day from each fermenter and studied as described in the following sections.

\section{Cell Growth Measurements}

Samples were taken once a day, diluted in sterile MilliQ water (Millipore Q-POD ${ }^{\mathrm{TM}}$ Advantage A10), plated on YPD medium (Glucose 2\%, Peptone 2\%, Yeast Extract 1\%, Agar $1.7 \%$ ) and lysine agar medium (Oxoid, England) plates using an automated spiral platter WASP II (Don Whitley. Scientific Limited, England), and incubated at $28^{\circ} \mathrm{C}$ for $48 \mathrm{~h}$. The YPD medium provided the total yeast counts, whereas the lysine agar medium only provided the non-Saccharomyces cell counts because $S$. cerevisiae cannot grow using lysine as a unique nitrogen source. Appropriate dilution plates were counted, and 20 colonies from the must before the inoculation and the beginning (density 1070 for Macabeo and 1090 for Merlot, both of them at day 1), middle (density between 1050 and 1040) and end (density below 1000, and residual sugars below $5 \mathrm{~g} / \mathrm{l}$ ) of the fermentation were randomly selected and purified on YPD plates for yeast identification.

\section{Yeast Identification}

The yeasts were identified based on the RFLPs of the PCRamplified ITS-5,8S rDNA region from the isolated colonies as described by Esteve-Zarzoso et al. (1999). The RFLP patterns of the yeast isolates were compared with those of the www.yeast-id.org (https://www.yeast-id.org/) based on the method described by Esteve-Zarzoso et al. (1999) and grouped to a known yeast species. Yeast identification was confirmed by sequencing the amplified D1/D2 domain of the 26S rDNA of representative colonies of each identified group as described by Kurtzman and Robnett (1998) and comparing this sequence with those of the type strains included in GenBank ${ }^{\circledR}$. Identification 
was considered appropriate with similarities higher than $99 \%$. The sequencing was performed by Macrogen.

Saccharomyces cerevisiae cells from the isolated colonies identified as $S$. cerevisiae were further characterized by Interdelta PCR analysis as described by Legras and Karst (2003).

\section{Massive Sequencing Analysis}

DNA (5-100 ng) was extracted from $1 \mathrm{ml}$ samples taken at the beginning, middle and end of the fermentation using the recommended procedure for the DNeasy Plant Mini kit (Qiagen, Hilden, Germany), including three bead-beating steps for 3 min in a FastPrep-24 bead beater (MP Bio, Solon, OH) to homogenize the samples. The extracted DNA was stored at $-20^{\circ} \mathrm{C}$ until further processing. A $350 \mathrm{bp}$ (on average) 18S rRNA gene fragment was amplified in triplicate from each DNA sample with the universal primers FR1 (5-ANCCATTCAATCGGTANT3) and FF390 (5-CGATAACGAACGAGACCT-3) (Chemidlin Prévost-Bouré et al., 2011). All primers had an Ion Torrent tag, and the universal primer included a 10-bp barcode unique to each amplified sample. The PCR reactions contained 5-100 ng DNA template, $1 \times$ GoTaq Green Master Mix (Promega), $1 \mathrm{mM}$ $\mathrm{MgCl}_{2}$, and $2 \mathrm{pmol}$ of each primer. The reaction conditions consisted of $94^{\circ} \mathrm{C}$ for $3 \mathrm{~min}$, followed by 35 cycles of $1 \mathrm{~min}$ at $94^{\circ} \mathrm{C}, 1 \mathrm{~min}$ at $52^{\circ} \mathrm{C}$ and $1 \mathrm{~min}$ at $72^{\circ} \mathrm{C}$, and a final extension phase for $10 \mathrm{~min}$ at $72^{\circ} \mathrm{C}$. The PCR products were pooled by sample and cleaned using a GeneRead Size Selection kit (Qiagen, Hilden, Germany). The cleaned PCR products were submitted to the Centre for Omic Sciences (Reus, Spain), where their quality was assessed with a Bionalyzer and their quantity was adjusted for sequencing. The raw sequences were demultiplexed and quality filtered using QIIME v1.8.0 (Caporaso et al., 2010a). Reads were discarded if the average quality score of the read was $<25$, if the length of the read was $<200$ or $>400$ and they contained one or more ambiguous base calls. Operational taxonomic units (OTUs) were assigned using QIIME's uclust-based (Edgar, 2010) open-reference OTU-picking workflow with a threshold of $97 \%$ pairwise identity. The OTU sequences were aligned using PYNAST (Caporaso et al., 2010b) against the SILVA 119 reference database (Pruesse et al., 2007). Taxonomic assignments were made in QIIME against the SILVA 119 database using the naive Bayesian classifier rdp (Wang et al., 2007). The template alignment of the Greengenes core set filtered at 97\% similarity. The OTU taxonomy was determined using the RDP classifier retrained toward the GreenGenes bacterial 16S rRNA database (13_8 release) (DeSantis et al., 2006) at 97\% similarity. Chimeric sequences were identified and removed using ChimeraSlayer (Haas et al., 2011), and a phylogenic tree was generated from the filtered alignment using FastTree (Price et al., 2009). A final OTU table was created that excluded excluding unaligned sequences and singletons (sequences observed just once). To avoid biases generated by differences in sequencing depth, the OTU table was rarified to an even depth of 20,000 sequences per sample in comparisons of all sample types in this study.

\section{PCR-DGGE}

The U1GC/U2 primers were used to amplify the specific U1/U2 domain of the $28 \mathrm{~S}$ ribosomal region of yeast (Meroth et al.,
2003). The PCR amplifications were performed on a Gene Amp PCR System 2700 (Applied Biosystems, Fosters City, USA) using EcoTaq DNA Polymerase (Ecogen, Spain). The Dcode universal mutation detection system (Bio-Rad, Hercules, Calif.) was used to run the DGGE analysis. The amplification of the fragments and denaturing electrophoresis were performed according to Meroth et al. (2003). The bands were excised from the gels, and the DNA was eluted overnight in $40 \mu \mathrm{l}$ of $10 \mathrm{mM}$ Tris $\mathrm{pH} 8$ and $1 \mathrm{mM}$ EDTA (TE) at $4{ }^{\circ} \mathrm{C}$. The DNA was re-amplified with the same pair of primers without the GC-clamp and sequenced by Macrogen. The BLASTN algorithm was applied to the GenBank database to identify sequences (http://www.ncbi.nlm.nih.gov/BLAST/). We considered appropriate the identification of the sequences with the corresponding type strains sequences when the sequence identity was higher than $98 \%$.

\section{Analysis of Volatile Compounds}

The aromatic compounds were extracted using adsorption and separate elution from an isolute ENV+ cartridge packed with $1 \mathrm{~g}$ of highly crosslinked styrene-divinyl benzene (SDVB) polymer (40-140 mm, cod. no. 915- 0100-C), as previously reported by Boido et al. (2003). The cartridges were sequentially equilibrated with methanol $(15 \mathrm{~mL})$ and distilled water $(20 \mathrm{~mL})$. A sample of $50 \mathrm{~mL}$ of wine diluted with $50 \mathrm{~mL}$ of distilled water and containing $0.1 \mathrm{~mL}$ of internal standard (1-heptanol at $230 \mathrm{mg} / \mathrm{L}$ in a $50 \%$ hydroalcoholic solution) was applied with an appropriate syringe $(4-5 \mathrm{~mL} / \mathrm{min})$, and the residue was washed with $15 \mathrm{~mL}$ of distilled water. The aromatic compounds were eluted with $30 \mathrm{~mL}$ of dichloromethane. The solution was dried with $\mathrm{Na}_{2} \mathrm{SO}_{4}$, concentrated to $1.5 \mathrm{~mL}$ on a Vigreux column, stored at $10^{\circ} \mathrm{C}$, and, immediately prior to GC-MS analysis, further concentrated to $150 \mu \mathrm{L}$ under a gentle nitrogen stream. The GC/MS analyses were conducted using a Shimadzu-QP 2010 ULTRA (Tokyo, Japan) mass spectrometer equipped with a Stabilwax $(30 \mathrm{~m} \times 0.25 \mathrm{~mm}$ i.d., $0.25 \mu \mathrm{m}$ film thickness $)$ (Restek) capillary column. The components of the wine aromatic compounds were identified comparing their linear retention indices with those of pure standards. (Aldrich, Milwaukee, $194 \mathrm{WI}$ ). The mass spectral fragmentation patterns were also compared with those stored in databases. GC-FID and GC-MS instrumental procedures using an internal standard (1-heptanol) were applied for quantitative purposes, as described previously by Boido et al. (2003). Ethanol and residual sugars were quantified using Winescan FT 120 (WineScan FT120 Type 77110, Foss Analytical, Denmark).

\section{Sensory Analysis}

A specialized panel (13 panelists) analyzed the sensorial attributes of Macabeo and Merlot wines fermented with $H$. vineae and $S$. cerevisiae. The wines were analyzed by means of a triangle test and descriptive analysis. The aim of the triangle test was to distinguish the wine fermented with $H$. vineae from the wine fermented with $S$. cerevisiae. The descriptive test emphasized the aroma and flavor attributes: Reduction, fresh fruit, candied fruit, flowery, aromatic plant, yeast, toasted (phenolic), herbaceous, aroma, sourness, structure, bitterness, volume and global impression. 


\section{Statistical Analysis}

The variance the aromatic compounds was analyzed using the Statistica 7.1 software (StatSoft, Tulsa, OK, 1984-2005). The sensory analysis results were submitted to Student's $t$-test. The results were considered significant when the associated $p$-value was below 0.05 .

\section{RESULTS}

\section{H. vineae and S. cerevisiae Fermentations}

The changes in the density and yeast populations during the alcoholic fermentations of both Macabeo and Merlot grapes are presented in Figure 1. The Macabeo must (Figure 1A) inoculated with $H$. vineae required a longer fermentation process (19 days) than those inoculated with $S$. cerevisiae (14 days) due to slower fermentation kinetics and a longer latency phase. However, Merlot grapes (Figure 1B) inoculated with $H$. vineae and with $S$. cerevisiae showed a similar fermentative progress, completing the fermentation in 9 days. This fact could be explained by the early presence of non-inoculated S. cerevisiae in the first stages of the fermentation.

No significant differences were observed in the ethanol concentration obtained at the end of the fermentation of both varieties (10.75 \pm 0.20 for Macabeo and $12.75 \pm 0.10$ for Merlot wines). Although all the wines were considered as "dry" (sugar concentration bellow 2 g residual sugars /L), a small difference was observed in the residual sugars in the Macabeo fermentation because the musts fermented with $H$. vineae left $1.7 \pm 0.3 \mathrm{~g}$ fructose/L, while all the other wines each of the residual sugars (glucose or fructose) were below $1 \mathrm{~g} / \mathrm{L}$.

The yeast population was quantified based on the colony growth on YPD and lysine agar medium. The total yeast population (YPD) was similar for the Macabeo and Merlot fermentations. The non-Saccharomyces yeasts counts (lysine agar) were slightly lower than the total yeast population counts in tanks inoculated with $H$. vineae for both grape musts. The Macabeo must was submitted to a vacuum filtration, which reduced the initial yeast population and resulted in yeast counts of $8.8 \times 10^{4} \mathrm{cfu} / \mathrm{ml}$ on YPD and $5.8 \times 10^{4}$ on lysine agar in must before inoculation.

\section{Yeast Biodiversity in Merlot and Macabeo Musts}

We identified only three yeast species in Macabeo must (Figures 2A,B), with Candida zemplinina being the main yeast species representing more than $80 \%$ of the yeast population. The other two yeast species identified were Hanseniaspora uvarum and Torulaspora delbrueckii. Of these, H. uvarum represented $12.50 \%$ of the total yeast population, whereas $T$. delbrueckii represented only $3.13 \%$ of the population. This distribution significantly differed in the yeast population recovered from Merlot must (Figures 2C,D). We identified up to eleven yeast species, with $C$. zemplinina and $H$. uvarum being the main species representing a percentage of 41 and $39 \%$ of the total yeast population, respectively. The low yeast diversity in Macabeo must may be due to the prefermentative filtration protocol, which reduces the yeast population. Moreover, during Merlot fermentation the must maintains contact with grape skins, which releases yeasts during the whole process. In both musts, only non-Saccharomyces yeasts were detected.

\section{Yeast Population Dynamics during Fermentation}

The yeast population dynamics during fermentation after the inoculation of $S$. cerevisiae or $H$. vineae was followed in yeast colonies grown on YPD plates based on PCR-DGGE, and the massive sequencing of the $18 \mathrm{~S}$ rRNA gene at the beginning, middle and end of the fermentation.

For the Macabeo fermentation, the inoculated strain accounted for $80-90 \%$ of the yeast population recovered from the plates 1 and 2 days after inoculation with $H$. vineae, whereas C. zemplinina had completely disappeared by the second day. $S$. cerevisiae was present at the beginning of fermentation, but at a very low proportion (Figure 2A). Nevertheless, the S. cerevisiae population began to increase from the middle to the end of the fermentation, accounting for $60 \%$ of the population at the middle of the fermentation and $100 \%$ at the end of the fermentation. From this population, up to three different $S$. cerevisiae strains could be identified by interdelta analysis, although QA23 was the most abundant at the end of the fermentation (more than $90 \%$, results not shown). Cross contamination between cellar vats is common in commercial cellars during vintage. Nevertheless, $H$. vineae represented $40 \%$ of the yeast population at the middle of the fermentation, which demonstrated the capacity of this yeast to dominate the native microbiota, and a high proportion of its population remained active after the middle of the fermentation. However, this yeast was not able to overcome the initial microbiota in Merlot fermentations inoculated with $H$. vineae (Figure 2C) and exhibited very low recovery on plates at the beginning and middle of the fermentation $(\approx 5-7 \%)$. Other non-Saccharomyces yeasts (H. uvarum, C. zemplinina, and Pichia occidentalis) outgrew $H$. vinae at these stages. The predominant yeast throughout the fermentation was a non-inoculated $S$. cerevisiae, which was recovered from plates and represented $50 \%$ of the total population at the beginning of the fermentation.

Macabeo and Merlot fermentations inoculated with $S$. cerevisiae showed similar yeast population patterns (Figures 2B,D). In both cases, the inoculated $S$. cerevisiae was able to rapidly dominate the fermentation because it was the only cultivable yeast recovered throughout the fermentation.

The PCR-DGGE profiles obtained for the DNA extracted directly from the wine during alcoholic fermentation identified the detected yeasts as $S$. cerevisiae, $H$. uvarum, $H$. vineae, and C. zemplinina (Table 1). S. cerevisiae was detected in all fermentations after the first day of inoculation, including in fermentations not inoculated with the commercial S. cerevisiae. Nevertheless, the $S$. cerevisiae in these latter fermentations appeared to be a different strain, as evidenced different migration patterns on DGGE gels. S. cerevisiae QA23 shows a particularity in PCR-DGGE because it produces a double band, which is not observed in other S. cerevisiae strains. All the bands excised from the gel migrating to the same height resulted in at least $99.9 \%$ sequence similarity to $S$. cerevisiae type strain. Merlot 


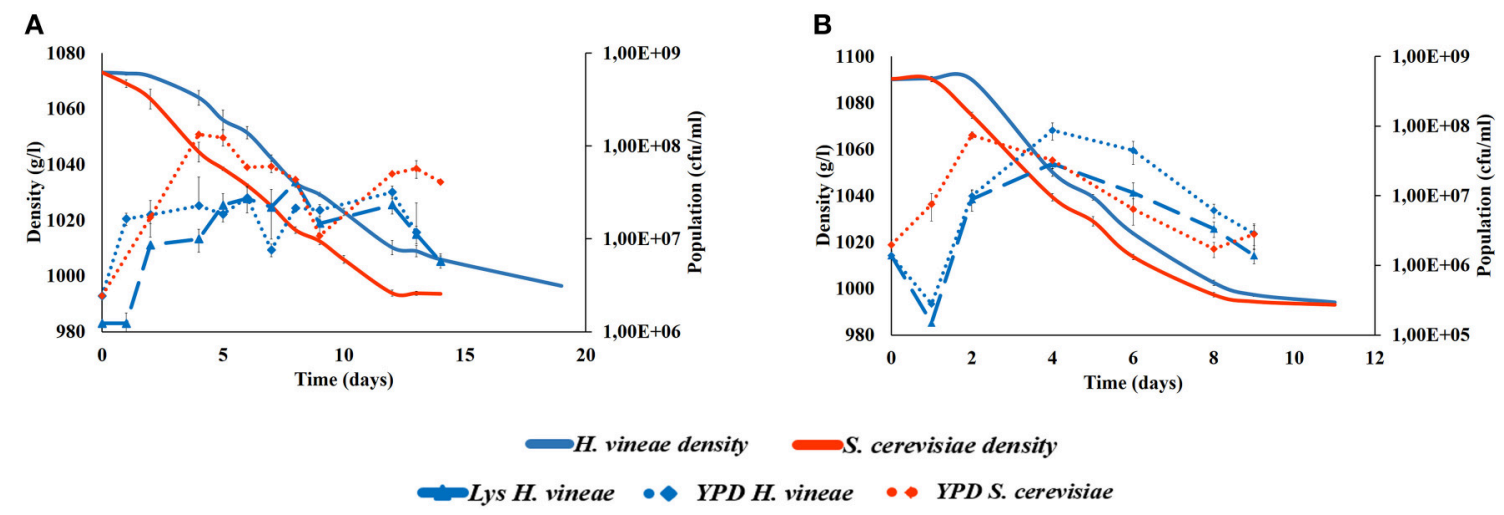

FIGURE 1 | Density measures of fermentations kinetics (-) and yeast population (cfu/ml) growth in YPD ( $)$ and lysine agar ( $(\Delta)$ medium of Macabeo (A) and Merlot (B) tanks inoculated with $H$. vineae (Blue) or S. cerevisiae (Red).

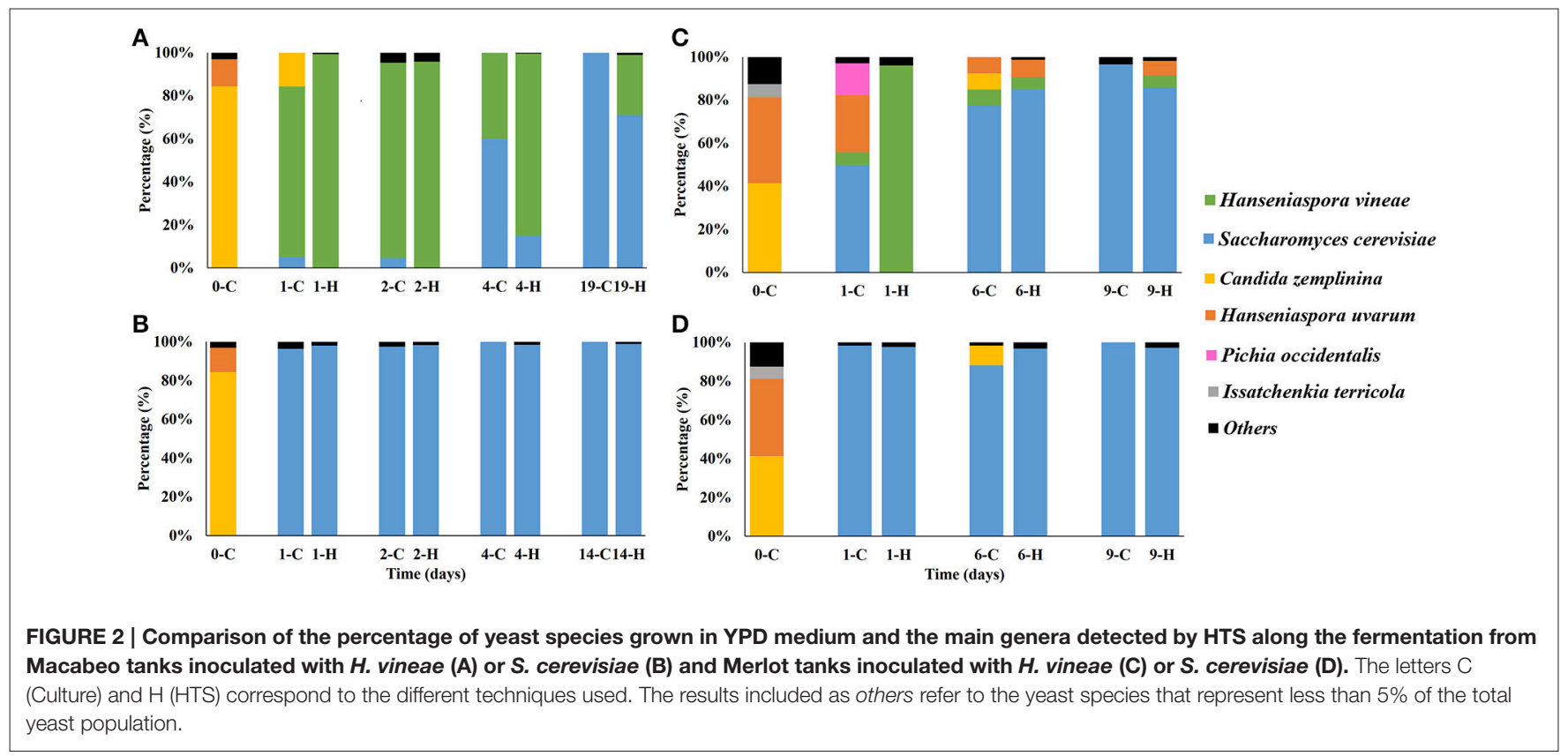

and Macabeo musts inoculated with $H$. vineae exhibited more yeast diversity at the beginning of the fermentations than musts inoculated with $S$. cerevisiae, and $H$. vineae was detected until the end of these fermentations.

A high-throughput sequencing (HTS) approach was also used to assess the fermented wine yeast biodiversity. After the removal of low quality sequences and those failing alignment, 642,105 18S rRNA amplicon sequences were generated from 9 Macabeo and 6 Merlot wine samples. The average number of sequences per sample was 42,807 , with an average length of $299 \mathrm{bp}$, and these sequences clustered into 16,302 operational taxonomic units (OTUs; 97\% nucleotide identity). To avoid diversity overestimation, singletons (sequences observed only once) were eliminated, and each sample was rarified to an even depth of 20,000 sequences to avoid biases generated by differences in sequencing depth. The number of different
OTUs was then reduced to 634, and 34 genera were identified. Good's coverage index was $99.7 \%$ on average, indicating that the global yeast diversity was mostly covered. The numbers of observed OTUs did not differ between Macabeo or Merlot wine samples inoculated with $S$. cerevisiae or $H$. vineae (Figure 3A). However, the number of genera was significantly higher at the beginning of the Merlot fermentation and tended to decrease toward the end of the fermentation, whereas the number of genera in Macabeo fermentation samples was lower than that in Merlot fermentations and relatively constant throughout the fermentation (Figure 3B). Most of the yeast population in all fermentations ( $97.7 \%$ on average) was represented by the inoculated $S$. cerevisiae and $H$. vineae strains (Tables $\mathbf{1}$, 2), whereas other non-Saccharomyces, such as H. uvarum and Zygosaccharomyces, accounted for only $1.9 \%$ of the sequences, and the remaining genera represented less than $0.5 \%$ of the 


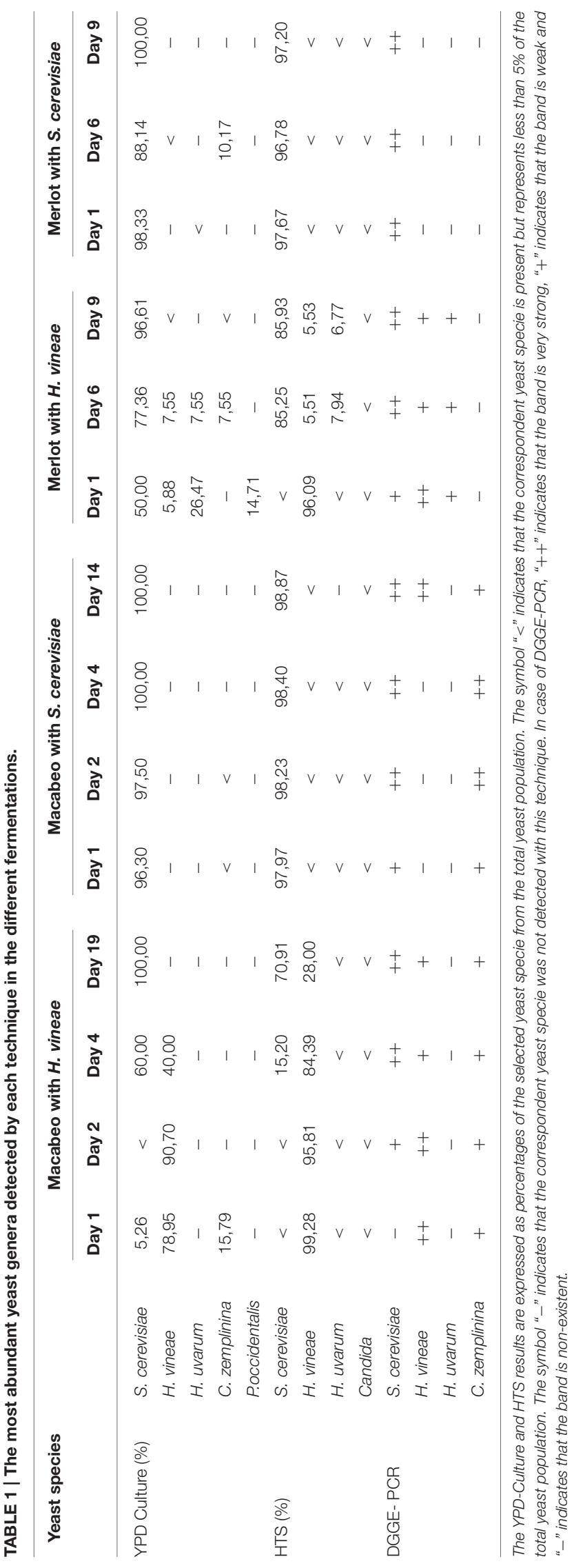

sequences (Table 2). Some of the detected fungi were not related to alcoholic fermentation (p.e. Aerobasidium, Aspergillus, Sporobolomyces); however, they were mainly detected at the beginning of the fermentation, and their populations quickly decreased or disappeared (Table 2). Interestingly, Dekkera was only detected in Merlot samples, and we were able to observe a small but distinct increase during the fermentations with both inocula.

\section{Volatile Compound Composition}

Fifty volatile compounds produced during alcoholic fermentations of natural Macabeo musts inoculated with $H$. vineae and S. cerevisiae were identified and quantified in the Macabeo wines. These compounds were classified into 10 groups, (acetates, acids, alcohols, C6 compounds, carbonyl compounds, esters, phenols, lactones, unusual compounds (named here as "rares") and terpenes). Table 3 shows the mean concentration of the identified volatile compounds. To assess the possible contribution of the different components to the wine aroma, the detection threshold and aroma descriptor reported in the literature are included for each compound.

Significant differences between yeasts were only observed in three of the 10 groups of compounds (Acetates and rares in Figure 4A and alcohols in Figure 4B).

Both yeasts primarily produced alcohols and esters, and three (isobutanol, isoamyl alcohol, and phenyl ethanol) of the eleven identified alcohols reached the threshold of perception reported in the literature. Of these three alcohols, phenyl ethanol provides good aromas that are described as rose and honey-like. Among the identified esters, ethyl hexanoate reached the threshold of perception and contributes a green apple aroma. The compounds constituting the next most abundant group produced by $H$. vineae are classified as rare and included $\mathrm{N}$-acetyl tyramine and $1 \mathrm{H}$-indole-3-ethanol acetate ester. These compounds were not found in the wine fermented with S. cerevisiae.

As shown in Table 3, a total of 7 acids were identified; hexanoic, decanoic and octanoic acid showed the highest concentration, and octanoic acid exceed the odor threshold reported in the literature.

Four acetates were identified, and phenethyl acetate was the most interesting. Specifically, this compound was 50 times more abundant in wines fermented with $H$. vineae than in those fermented by $S$. cerevisiae. This compound endows wine with floral, fruity and honey-like aromas.

Six phenolic compounds were identified, as shown in Table 3. These compounds did not reach the threshold of detection, and their contribution to wine aroma is consequently expected to be insignificant. One of these compounds, 4-ethylguaiacol, is generally attributed to the presence of Brettanomyces, although it was identified in wines fermented with $H$. vineae.

Six terpenes were identified, as shown in Table 3. The concentrations of these compounds were lower than the threshold, and they are consequently not expected to contribute to the wine flavor profiles.

\section{Sensory Analysis}

To evaluate the ability of $H$. vineae to produce a wine with attributes that differ from those of a wine fermented with $S$. 
TABLE 2 | Percentage of main genera and species detected by HTS after the inoculation of $\boldsymbol{H}$. vineae or S. cerevisiae on Merlot and Macabeo wines.

\begin{tabular}{|c|c|c|c|c|c|c|c|c|c|c|c|c|c|c|c|}
\hline \multirow[t]{2}{*}{ Days from inoculation } & \multicolumn{5}{|c|}{ Macabeo H.vineae } & \multicolumn{4}{|c|}{ Macabeo S. cerevisiae } & \multicolumn{3}{|c|}{ Merlot $\boldsymbol{H}$. vineae } & \multicolumn{3}{|c|}{ Merlot S. cerevisiae } \\
\hline & 1 & 2 & 4 & 14 & 19 & 1 & 2 & 4 & 14 & 1 & 6 & 8 & 1 & 6 & 8 \\
\hline Saccharomyces cerevisiae & 0.47 & 3.68 & 15.20 & 60.32 & 70.91 & 97.97 & 98.23 & 98.40 & 98.87 & 0.44 & 85.25 & 85.93 & 97.67 & 96.78 & 97.20 \\
\hline Hanseniaspora vineae & 99.28 & 95.81 & 84.39 & 38.75 & 28.00 & 0.64 & 0.48 & 0.57 & 0.24 & 96.09 & 5.51 & 5.53 & 0.53 & 1.69 & 1.60 \\
\hline Hanseniaspora uvarum & 0.07 & 0.07 & 0.01 & $<$ & 0.01 & 0.05 & 0.02 & 0.01 & $<$ & 0.33 & 7.94 & 6.77 & 0.24 & 0.40 & 0.15 \\
\hline Zygosaccharomyces & 0.01 & 0.05 & 0.23 & 0.47 & 0.60 & 0.67 & 0.66 & 0.52 & 0.39 & 0.41 & 0.49 & 0.49 & 0.53 & 0.51 & 0.47 \\
\hline Saccharomyces (others) & 0.01 & 0.03 & 0.08 & 0.31 & 0.39 & 0.55 & 0.54 & 0.43 & 0.44 & $<$ & 0.33 & 0.36 & 0.36 & 0.37 & 0.36 \\
\hline Aureobasidium & $<$ & $<$ & - & 0.01 & - & - & - & - & - & 1.83 & 0.03 & 0.02 & 0.33 & 0.05 & 0.05 \\
\hline Candida & 0.09 & 0.14 & 0.03 & 0.06 & 0.07 & 0.11 & 0.07 & 0.06 & 0.05 & 0.31 & 0.18 & 0.46 & 0.19 & 0.18 & 0.12 \\
\hline Pichia & 0.05 & 0.17 & 0.05 & $<$ & 0.01 & - & - & $<$ & - & 0.03 & 0.26 & 0.26 & 0.01 & 0.01 & - \\
\hline Dekkera & - & - & - & - & - & - & - & - & - & - & $<$ & 0.17 & - & - & 0.03 \\
\hline Aspergillus & - & 0.01 & - & - & - & - & - & - & - & 0.11 & $<$ & 0.01 & 0.03 & $<$ & - \\
\hline Sporobolomyces & - & - & - & - & - & - & - & - & - & 0.08 & $<$ & - & 0.02 & - & - \\
\hline Issatchenkia & 0.01 & 0.02 & 0.01 & 0.01 & 0.01 & 0.01 & - & $<$ & - & 0.02 & 0.01 & - & 0.01 & - & - \\
\hline Cryptococcus & - & $<$ & - & $<$ & - & - & - & - & - & 0.07 & - & - & 0.01 & - & - \\
\hline Diplodia & - & - & - & - & - & - & - & - & - & 0.07 & - & - & $<$ & - & - \\
\hline Zygoascus & - & - & - & - & - & $<$ & - & - & - & 0.03 & - & - & 0.02 & 0.01 & 0.01 \\
\hline Rhizina & - & - & - & - & - & - & - & - & - & 0.04 & - & - & 0.01 & - & - \\
\hline Catenulostroma & - & - & - & - & - & - & - & - & - & 0.05 & - & - & - & - & - \\
\hline Bensingtonia & - & - & - & 0.04 & - & - & - & - & - & $<$ & - & - & - & - & - \\
\hline Saccharomycodes & - & - & $<$ & 0.02 & 0.01 & - & - & - & $<$ & - & - & - & - & - & 0.01 \\
\hline Scheffersomyces & - & - & - & - & - & - & - & - & - & 0.02 & $<$ & $<$ & 0.01 & - & - \\
\hline Wickerhamomyces & 0.01 & 0.01 & $<$ & - & - & - & - & - & - & 0.01 & - & - & - & - & - \\
\hline Cladosporium & - & - & - & - & - & - & - & - & - & 0.01 & - & - & 0.01 & - & - \\
\hline Sugiyamaella & 0.01 & 0.01 & $<$ & - & - & - & - & - & - & - & - & - & - & - & - \\
\hline Trigonopsis & 0.01 & 0.01 & - & - & $<$ & - & - & - & - & - & - & - & - & - & - \\
\hline Lipomyces & - & - & - & - & - & - & - & - & - & - & 0.01 & 0.01 & - & - & - \\
\hline Phillipsia & - & - & - & - & - & - & - & - & - & 0.01 & - & - & - & - & - \\
\hline Wallemia & 0.01 & - & - & - & - & $<$ & - & - & - & $<$ & - & - & - & - & - \\
\hline Vanderwaltozyma & - & - & - & - & - & - & - & - & $<$ & - & $<$ & - & - & - & $<$ \\
\hline Cochliobolus & - & - & - & - & - & - & - & - & - & 0.01 & - & - & - & - & - \\
\hline Malassezia & - & - & - & - & - & $<$ & - & - & - & $<$ & - & $<$ & - & - & - \\
\hline Bispora & - & - & - & - & - & - & - & - & - & $<$ & - & - & $<$ & - & - \\
\hline Rhodotorula & - & - & - & - & - & - & - & - & - & $<$ & - & - & $<$ & - & - \\
\hline Metschnikowia & - & - & - & - & - & - & - & - & - & $<$ & $<$ & - & - & - & - \\
\hline Phoma & - & - & - & - & - & - & - & - & - & $<$ & - & $<$ & - & - & - \\
\hline Agaricostilbum & - & - & - & - & - & - & - & - & - & $<$ & - & $<$ & - & - & - \\
\hline Baudoinia & - & - & - & - & - & - & - & - & - & $<$ & - & - & - & - & - \\
\hline
\end{tabular}

The symbol "<" indicates percentages values lower than 0.01 and bigger than 0 . The symbol "- "indicates not detected by HTS.

cerevisiae, the produced wines were analyzed with triangle and descriptive tests.

In the triangle test of Macabeo wine (Figure 5), wine-tasters easily distinguished the wine fermented with $H$. vineae from that fermented with $S$. cerevisiae, and the majority selected the wine fermented with $H$. vineae as their preference. In the descriptive test, the wine fermented with $H$. vineae received the best rating. Notably, wine fermented by $H$. vineae showed a significantly stronger flowery aroma profile $(p=0.037)$ than wine produced with S. cerevisiae.

The Merlot must could not be evaluated as a consequence of a powerful reduction note that could not be corrected for the tasting.

\section{DISCUSSION}

In this work, we performed semi-industrial fermentations with $H$. vineae and S. cerevisiae using Macabeo and Merlot musts in order to evaluate the differences in yeast populations during fermentation and the wines produced. We followed the yeast population dynamics in both grape musts inoculated with $H$. vineae and $S$. cerevisiae by plate culturing on YPD medium, PCR-DGGE with yeast general primers, as well as yeast general primers and the HTS of $18 \mathrm{~S}$ rRNA gene.

As expected, the fermentation of musts inoculated with $H$. vineae required more time than fermentations inoculated with S. cerevisiae, demonstrating the high fermentative ability of this 
TABLE 3 | Average concentrations of the two fermentations ( \pm Standard Deviation) in $\mu \mathrm{g} / \mathrm{l}$.

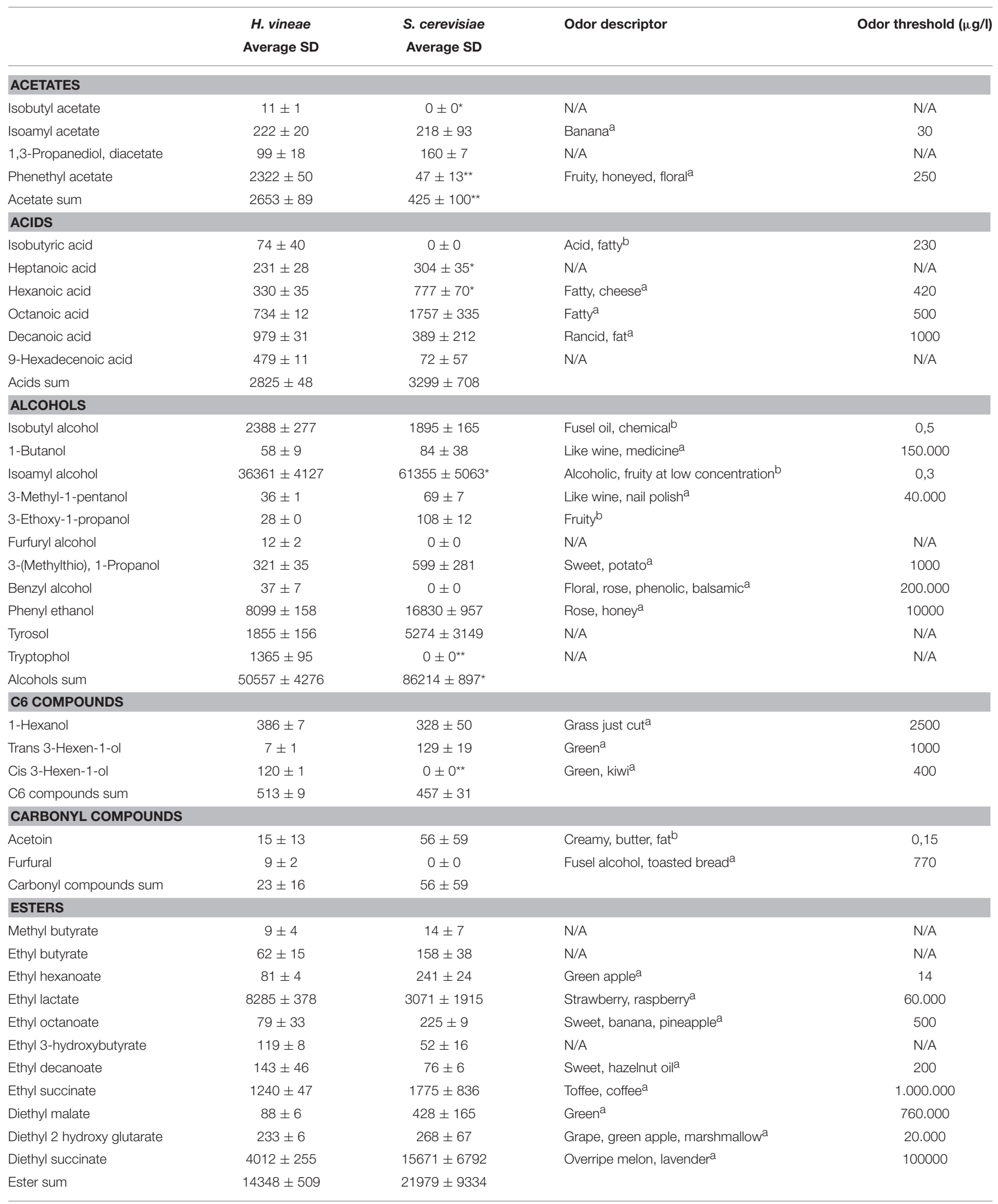


TABLE 3 | Continued

\begin{tabular}{|c|c|c|c|c|}
\hline & $\begin{array}{c}\text { H. vineae } \\
\text { Average SD }\end{array}$ & $\begin{array}{l}\text { S. cerevisiae } \\
\text { Average SD }\end{array}$ & Odor descriptor & Odor threshold $(\mu \mathrm{g} / \mathrm{l})$ \\
\hline \multicolumn{5}{|l|}{ PHENOLS } \\
\hline 4-ethylguaiacol & $73 \pm 66$ & $0 \pm 0$ & Bretty flavors ${ }^{a}$ & 110 \\
\hline 4-vinylguaiacol & $33 \pm 21$ & $28 \pm 14$ & Clove, curry ${ }^{a}$ & 40 \\
\hline Acetovainillone & $14 \pm 5$ & $15 \pm 13$ & $\mathrm{~N} / \mathrm{A}$ & $\mathrm{N} / \mathrm{A}$ \\
\hline Phenol sum & $183 \pm 41$ & $188 \pm 11$ & & \\
\hline \multicolumn{5}{|l|}{ LACTONES } \\
\hline Butyrolactone & $223 \pm 1$ & $251 \pm 6$ & Toasted burned ${ }^{a}$ & 1000 \\
\hline 5-carboethoxy-gamma-butyrolactone & $127 \pm 7$ & $76 \pm 11$ & $\mathrm{~N} / \mathrm{A}$ & $\mathrm{N} / \mathrm{A}$ \\
\hline 1H-Indole-3-ethanol, acetate (ester) & $1377 \pm 8$ & $0 \pm 0^{* *}$ & $\mathrm{~N} / \mathrm{A}$ & $\mathrm{N} / \mathrm{A}$ \\
\hline Rare sum & $3417 \pm 4$ & $0 \pm 0^{\star \star \star}$ & & \\
\hline \multicolumn{5}{|l|}{ TERPENES } \\
\hline Linalool & $12 \pm 2$ & $28 \pm 13$ & Rose $^{a}$ & 50 \\
\hline Alpha-terpineol & $112 \pm 31$ & $0 \pm 0$ & Floral, pine ${ }^{a}$ & 400 \\
\hline Citronellol & $27 \pm 6$ & $39 \pm 5$ & Sweet, floral ${ }^{\mathrm{b}}$ & 18 \\
\hline Terpene sum & $150 \pm 23$ & $67 \pm 18$ & & \\
\hline
\end{tabular}

Odor descriptor and odor thresholds reported in the literature are included.

${ }^{*},{ }^{* \star},{ }^{\star \star \star}$ indicate significance at $p<0.05, p<0.01, p<0.001$ respectively.

a Fariña et al. (2015).

${ }^{b}$ Boido (2002).

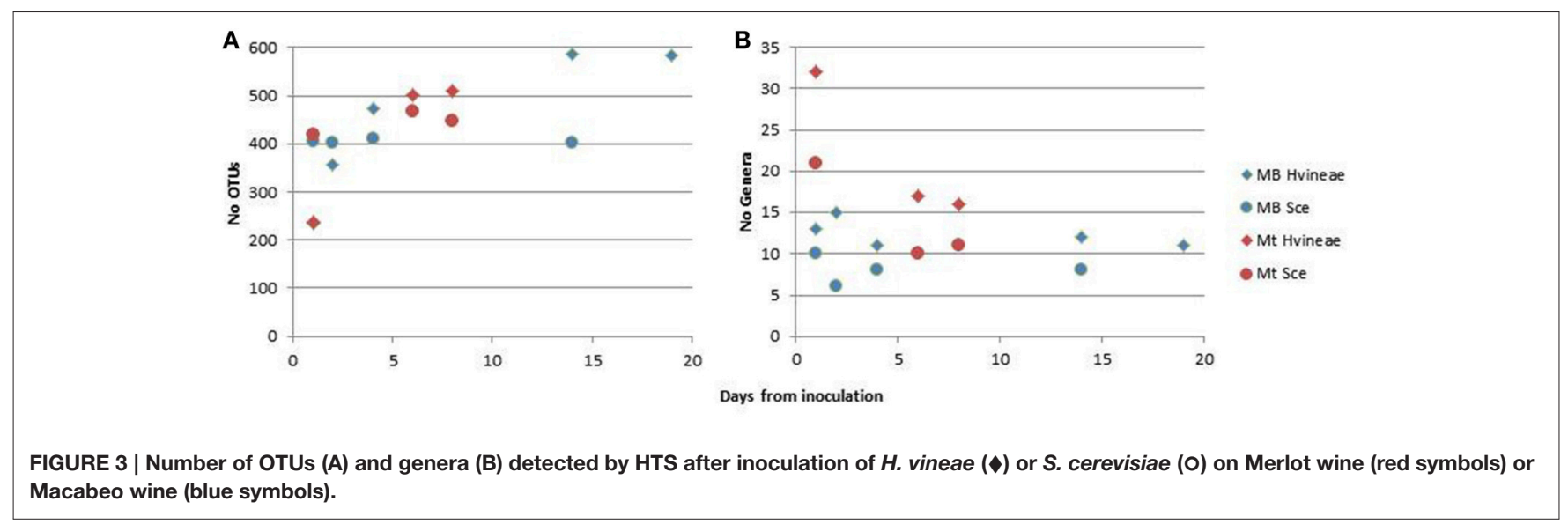

wine yeast with respect to $H$. vineae. However, rapid fermentation might not be a desired characteristic in the production of quality wines, due to flavor lost or high energetic demand for refrigeration (Medina et al., 2013).

The initial yeast diversity of the must was only analyzed after plate culturing. Before the inoculation, Merlot must presented a higher yeast diversity than Macabeo must, as evidenced by up to eleven different yeast species that were identified in the red variety, whereas the Macabeo contained only three species $(C$. zemplinina, H. uvarum, and T. delbrueckii). As expected, only non-Saccharomyces yeasts were recovered by cultivation from both musts before the inoculation because $S$. cerevisiae is not present in relevant amounts in grapes and is mostly associated with cellar equipment (Pretorius, 2000; Torija et al., 2001; Beltran et al., 2002).

The PCR-DGGE analysis identified $S$. cerevisiae and $H$. vineae as the main yeasts in both the Macabeo and Merlot fermentations. C. zemplinina was found only in Merlot, and these 

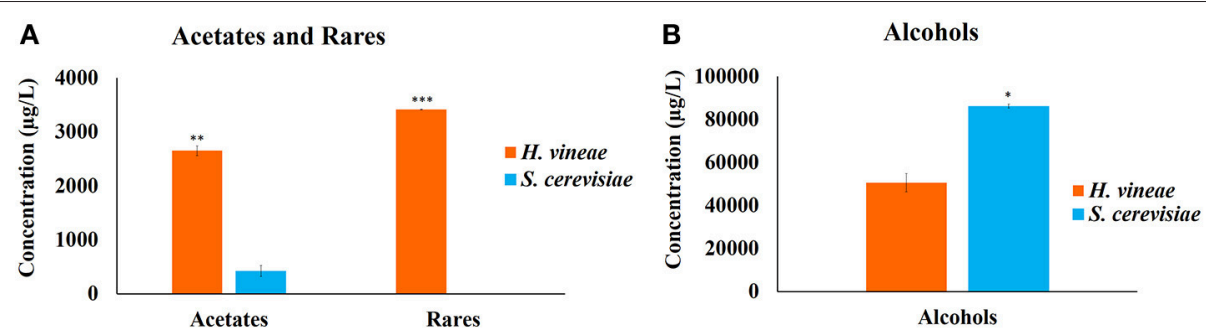

FIGURE 4 | Sum of compounds with significant differences produced by $\boldsymbol{H}$. vineae and S. cerevisiae (A) acetates and compounds listed as rare ( $\mathbf{N}$-acetyltyramine and $\mathbf{1 H}$-indole-3-ethanol acetate ester) (B) Alcohols. Code: ${ }^{\star, * *},{ }^{* \star \star}$ indicate significance at $p<0.05, p<0.01, p<0.001$, respectively.

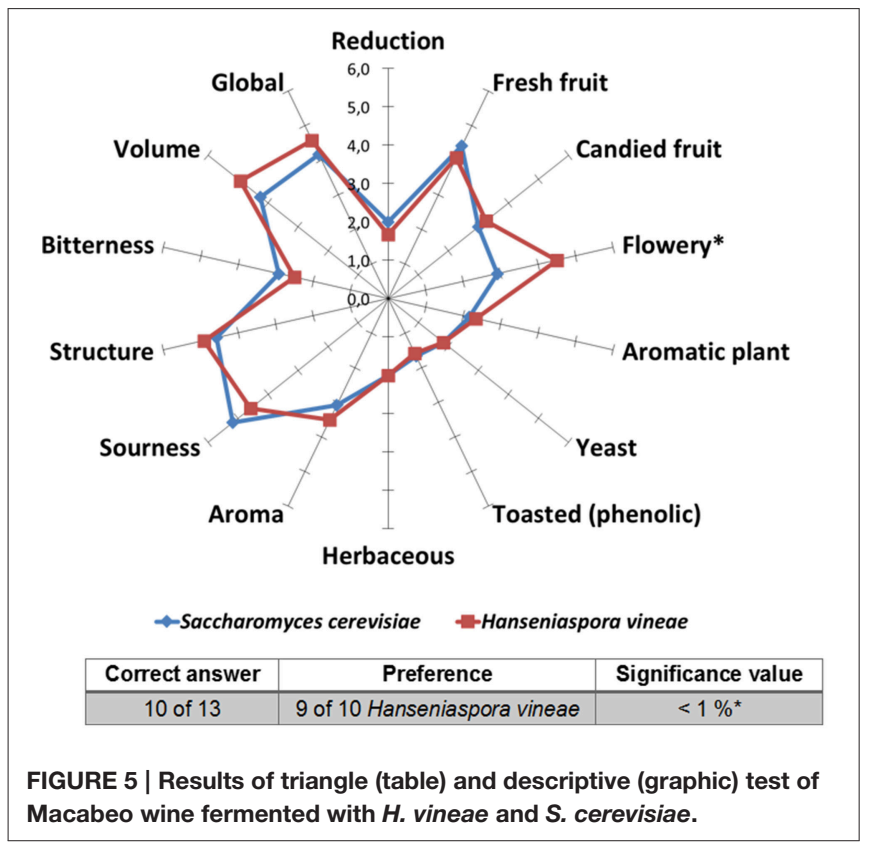

results corroborated those observed after the plate culture. Other minor yeast species were not detected by PCR-DGGE, especially if their population densities were below $10^{3}-10^{4} \mathrm{CFU} / \mathrm{ml}$ or if their abundance was two orders of magnitude lower than that of the main species, as reported in previous studies (Mills et al., 2002; Prakitchaiwattana et al., 2004; Andorrà et al., 2008).

Even if must samples were not included in the HTS approach, this technique clearly detected higher levels of fungal diversity than the other techniques. Specifically, a total of 32 genera with a great diversity of OTUs were identified within each genus. The HTS technique was also able to detect yeast genera not related with fermentation, and some of these yeasts are associated with spoilage (like Dekkera/Brettanomyces). Although the proportion of these yeasts was very low, the changes in their proportion throughout the fermentation suggested that they were active and represented a potential risk for the spoilage of the final wine. Thus, the HTS technique confirmed the general trend obtained for the most abundant yeast populations by plate culturing and PCR-DGGE, but it also facilitated the detection and tracking of some minor yeast genera that may significantly impact the quality of the wine.
The culturing, PCR-DGGE and HTS analysis confirmed a decrease in the yeast genera diversity from the beginning to the end of fermentations, and these techniques also consistently indicated that the yeast diversity was higher in Merlot fermentations than in Macabeo fermentations. The low diversity exhibited by Macabeo must before inoculation may be a consequence of its treatment with a vacuum filter. The objective of this treatment was to clean the must and remove solid and colloidal particles, but it also reduced autochthonous yeasts and nutrients in the must. We used this protocol for two reasons: to clean the Macabeo must and to remove colloidal and solid particles and also it was affected by rain and exhibited some spoilage. Thus, we wanted to reduce the autochthonous yeast population because we planned to inoculate the must with $H$. vineae. We achieved these objectives. Furthermore, the Merlot was selectively handpicked in order to obtain the healthiest bunches of grapes. The results from plate culturing, PCR-DGGE and HTS indicate that $H$. vineae was able to overcome the autochthonous microbiota in the Macabeo must, constituting a high proportion of the yeast population until the middle of the fermentation and showing good fermentative capacity. However, $H$. vineae represented a very low proportion of the yeast population in Merlot must after the inoculation. However, after the inoculation (day 1), the percentages of the identified yeasts were different based on the method of estimation, being the population of $H$. vineae hardly recovered on plates. S. cerevisiae was the most abundant yeast recovered from plates, whereas it was present at much lower levels in all cultureindependent methods (HTS and DGGE). This observation could be related to the well-reported interaction between $S$. cerevisiae and non-Saccharomyces yeasts during wine fermentation: nonSaccharomyces yeasts are quickly displaced by S. cerevisiae, which might kill or at least result in viable but not cultivable (VBNC) statuses, as indicated in several recent reports (Millet and Lonvaud-Funel, 2000; Pérez-Nevado et al., 2006; Andorrà et al., 2010, 2011; Wang et al., 2015). However, we should emphasize that these culture-independent techniques also detect DNA from dead cells, which could also be the case. At later fermentation time points, all methods again produced coincident results and identified S. cerevisiae as the main population. Interestingly, the dominant $S$. cerevisiae was not the inoculated strain, suggesting that a cellar-resident strain took over. Furthermore, Merlot grapes are among the latest in the harvest in this cellar, and, thus, the environmental contamination of the cellar is already 
high. The S. cerevisiae population began to increase and became the dominant species according to HTS and produced the most intense band profile of DGGE, and this unique yeast was recovered at the end of the fermentation.

The final wine obtained by fermenting Macabeo must with $H$. vineae was preferred over the wine fermented with $S$. cerevisiae for its notable fruity and flowery aroma. This result corroborates those of studies that performed mixed fermentations with $H$. vineae and obtained high amounts of an acetate ester, phenethyl acetate, which is responsible of the fruity and flowery aroma of wine (Viana et al., 2009, 2011). The chemical analysis revealed that wines inoculated with $H$. vineae contained 50 times more phenethyl acetate than wines inoculated with $S$. cerevisiae, which explains the results of our sensory analysis and agrees with previous observations (Medina et al., 2013).

The production of $\mathrm{N}$-acetyltyramine and $1 \mathrm{H}$-indole-3ethanol acetate ester also differed. These compounds were abundant in wines inoculated with $H$. vineae and could not be detected in wines fermented with $S$. cerevisiae. These compounds could be derived from tyrosol, and this hypothesis is supported by the high concentrations of tyrosol in wines inoculated with S. cerevisiae. This difference could be explained by the production of unusual compounds from tyrosol in wines inoculated with $H$. vineae. However, aromatic descriptors associated with these compounds have not yet been reported.

\section{CONCLUSION}

Interest in the use of non-Saccharomyces yeasts in winemaking has been increasing. $H$. vineae is an apiculate non-Saccharomyces yeast that has demonstrated a good fermentative rate in Macabeo must and resulted in more flowery wines, which is likely related to the higher production of phenylethyl acetate. However, the need for inoculation with $S$. cerevisiae must be emphasized because

\section{REFERENCES}

Andorrà, I., Berradre, M., Rozès, N., Mas, A., Guillamón, J. M., and EsteveZarzoso, B. (2010). Effect of pure and mixed cultures of the main wine yeast species on grape must fermentations. Eur. Food Res. Technol. 231, 215-224. doi: 10.1007/s00217-010-1272-0

Andorrà, I., Landi, S., Mas, A., Guillamón, J. M., and Esteve-Zarzoso, B. (2008). Effect of enological practices on microbial populations using culture-independent techniques. Food Microbiol. 25, 849-856. doi: 10.1016/j.fm.2008.05.005

Andorrà, I., Monteiro, M., Esteve-Zarzoso, B., Albergaria, H., and Mas, A. (2011). Analysis and direct quantification of Saccharomyces cerevisiae and Hanseniaspora guilliermondii populations during alcoholic fermentation by fluorescence in situ hybridisation, flow cytometry and quantitative PCR. Food Microbiol. 28, 1483-1491. doi: 10.1016/j.fm.2011.08.009

Beltran, G., Torija, M. J., Novo, M., Ferrer, N., Poblet, M., Guillamón, J. M., et al. (2002). Analysis of yeast populations during alcoholic fermentation: a six year follow-up study. Syst. Appl. Microbiol. 25, 287-293. doi: 10.1078/0723-202000097

Boido, E. (2002). Modificaciones Producidas por la Fermentación Maloláctica en la Fracción Aromática de los Vinos Tannat. Ph.D., thesis. Universidad de la República, Facultad de Química, Montevideo, Uruguay.

Boido, E., Lloret, A., Medina, K., Fariña, L., Carrau, F., Versini, G., et al. (2003). Aroma composition of Vitis vinifera cv. Tannat: the typical red
$H$. vineae is unable to finish the alcoholic fermentation. We did not use a $S$. cerevisiae strain in the inoculations with $H$. vineae, and the end of fermentation was consequently improperly controlled. Furthermore, the use of this yeast requires very healthy grape musts and is not recommended to use with grapes with a high and diverse yeast population or red musts, in which maceration with skins may be a significant source of yeast. In addition, the present study shows that the HTS technique detected not only the most abundant yeast populations obtained by plate culturing and PCR-DGGE but also some minor yeast genera that may significantly affect the quality of the wine.

\section{AUTHOR CONTRIBUTIONS}

JL: Performing the experiments, Design of experiments, Writing of the manuscript, Discussion of results, Performing Next Generation Sequencing, Analysis of results. VM: Performing the experiments, Design of experiments, Analysis of results, Discussion of results, Writing of the manuscript. MP: Performing Next Generation Sequencing, Analysis of results, Writing of the manuscript. FC: Design of experiments, Analysis of results, Discussion of results. GB: Design of experiments, Analysis of results, Discussion of results. AM: Design of experiments, Discussion of results, Analysis of results, Writing of the manuscript.

\section{ACKNOWLEDGMENTS}

MP is the recipient of a Beatriu de Pinòs Postdoctoral Fellowship with the support of the Ministry of Universities and Research of the Department of Economy and Knowledge of the Generalitat of Catalonia and the program COFUND Marie Curie Actions of the 7th Framework Program for R+D of EU.

wine from Uruguay. J. Agric. Food Chem. 51, 5408-5413. doi: 10.1021/jf03 $0087 \mathrm{i}$

Cabtree, H. G. (1929). Observations on the carbohydrate metabolism of tumours. Biochem. J. 23, 536-545. doi: 10.1042/bj0230536

Caporaso, J. G., Bittinger, K., Bushman, F. D., DeSantis, T. Z., Andersen, G. L., and Knight, R. (2010b). PyNAST: a flexible tool for aligning sequences to a template alignment. Bioinformatics 26, 266-267. doi: 10.1093/bioinformatics/ btp636

Caporaso, J. G., Kuczynski, J., Stombaugh, J., Bittinger, K., Bushman, F. D., Costello, E. K., et al. (2010a). QIIME allows analysis of highthroughput community sequencing data. Nat. Methods 7, 335-336. doi: $10.1038 /$ nmeth.f.303

Chemidlin Prévost-Bouré, N., Christen, R., Dequiedt, S., Mougel, C., Lelièvre, M., Jolivet, C., et al. (2011). Validation and application of a PCR primer set to quantify fungal communities in the soil environment by real time quantitative PCR. PLoS ONE 6:e24166. doi: 10.1371/journal.pone.0024166

DeSantis, T. Z., Hugenholtz, P., Larsen, N., Rojas, M., Brodie, E. L., Keller, K., et al. (2006). Greengenes, a chimera-checked 16S rRNA gene database and workbench compatible with ARB. Appl. Environ. Microb. 72, 5069-5072. doi: 10.1128/AEM.03006-05

Edgar, R. C. (2010). Search and clustering orders of magnitude faster than BLAST. Bioinformatics 26, 2460-2461. doi: 10.1093/bioinformatics/btq461

Esteve-Zarzoso, B., Belloch, C., Uruburu, F., and Querol, A. (1999). Identification of yeasts by RFLP analysis of the 5.8 S rRNA gene and the two ribosomal internal 
transcribed spacers. Int. J. Syst. Bacteriol. 49, 329-337. doi: 10.1099/0020771349-1-329

Fariña, L., Villar, V., Ares, G., Carrau, F., Dellacassa, E., and Boido, E. (2015). Volatile composition and aroma profile of Uruguayan Tannat wines. Food Res. Int. 69, 244-255. doi: 10.1016/j.foodres.2014.12.029

Fleet, G. H. (2008). Wine yeasts for the future. FEMS Yeast Res. 8, 979-995. doi: 10.1111/j.1567-1364.2008.00427.x

Haas, B. J., Gevers, D., Earl, A. M., Feldgarden, M., Ward, D. V., Giannoukos, G., et al. (2011). Chimeric 16S rRNA sequence formation and detection in Sanger and 454-pyrosequenced PCR amplicons. Genome Res. 21, 494-504. doi: $10.1101 /$ gr.112730.110

Jolly, N. P., Varela, C., and Pretorius, I. S. (2014). Not your ordinary yeast: nonSaccharomyces yeasts in wine production uncovered. FEMS Yeast Res. 14, 215-237. doi: 10.1111/1567-1364.12111

Kurtzman, C. P., and Robnett, C. J. (1998). Identification and phylogeny of ascomycetous yeasts from analysis of nuclear large subunit (26S) ribosomal DNA partial sequences. Antonie van Leeuwenhoek 73, 331-371. doi: 10.1023/A:1001761008817

Legras, J. L., and Karst, F. (2003). Optimisation of interdelta analysis for Saccharomyces cerevisiae strain characterization. FEMS Microbiol. Lett. 221, 249-255. doi: 10.1016/S0378-1097(03)00205-2

Maturano, P. Y., Rodríguez Assaf, L. A., Toro, E. M., Nally, C. M., Vallejo, M., Castellanos de Figueroa, L. I., et al. (2012). Multi-enzyme production by pure and mixed cultures of Saccharomyces and non-Saccharomyces yeasts during wine fermentation. Int. J. Food Microbiol. 155, 43-50. doi: 10.1016/j.ijfoodmicro.2012.01.015

Medina, K., Boido, E., Fariña, L., Gioia, O., Gomez, M. E., Barquet, M., et al. (2013). Increased flavour diversity of Chardonnay wines by spontaneous fermentation and co-fermentation with Hanseniaspora vineae. Food Chem. 141, 2513-2521. doi: 10.1016/j.foodchem.2013.04.056

Meroth, C. B., Hammes, W. P., and Hertel, C. (2003). Identification and population dynamics of yeasts in Sourdough fermentation processes by using PCR-denaturing gradient gel electrophoresis. Appl. Environ. Microbiol. 69, 7453-7461. doi: 10.1128/AEM.69.12.7453-7461.2003

Millet, V., and Lonvaud-Funel, A. (2000). The viable but non-culturable of wine micro-organisms during storage. Lett. Appl. Microbiol. 30, 136-141. doi: 10.1046/j.1472-765x.2000.00684.x

Mills, D. A., Johannsen, E. A., and Cocolin, L. (2002). Yeast diversity and persistence in botrytis-affected wine fermentations. Appl. Environ. Microbiol. 68, 4884-4893. doi: 10.1128/AEM.68.10.4884-4893.2002

Pérez-Nevado, F., Albergaria, H., Hogg, T., and Girio, F. (2006). Cellular death of two non-Saccharomyces wine related yeasts during mixed fermentation with Saccharomyces cerevisiae. Int. J. Food Microbiol. 108, 336-345. doi: 10.1016/j.ijfoodmicro.2005.12.012

Prakitchaiwattana, C. J., Fleet, G. H., and Heard, G. M. (2004). Application and evaluation of denaturing gradient gel electrophoresis to analyse the yeast ecology of wine grapes. FEMS Yeast Res. 4, 865-877. doi: 10.1016/j.femsyr.2004.05.004

Pretorius, I. S. (2000). Tailoring wine yeast for the new millennium: novel approaches to the ancient art of winemaking. Yeast 16,
675-729. doi: 10.1002/1097-0061(20000615)16:8<675::AID-YEA585>3. $0 . \mathrm{CO} ; 2-\mathrm{B}$

Price, M. N., Dehal, P. S., and Arkin, A. P. (2009). FastTree: computing large minimum evolution trees with profiles instead of a distance matrix. Mol. Biol. Evol. 26, 1641-1650. doi: 10.1093/molbev/msp077

Pruesse, E., Quast, C., Knittel, K., Fuchs, B. M., Ludwig, W., Peplies, J., et al. (2007). SILVA: a comprehensive online resource for quality checked and aligned ribosomal RNA sequence data compatible with ARB. Nucleic Acids Res. 35, 7188-7196. doi: 10.1093/nar/gkm864

Strauss, M. L. A., Jolly, N. P., Lambrechts, M. G., and van Rensburg, P. (2001). Screening for the production of extracellular hydrolytic enzymes by nonSaccharomyces wine yeasts. J. Appl. Microbiol. 91, 182-190. doi: 10.1046/j.13652672.2001.01379.x

Swangkeaw, J., Vichitphan, S., Butzke, C. E., and Vichitphan, K. (2011). Characterization of $\beta$-glucosidases from Hanseniaspora sp. and Pichia anomala with potentially aroma-enhancing capabilities in juice and wine. World J. Microbiol. Biotechnol. 27, 423-430. doi: 10.1007/s11274-010-0474-8

Torija, M. J., Rozès, N., Poblet, M., Guillamón, J. M., and Mas, A. (2001). Yeast population dynamics in spontaneous fermentations: comparison between two different wine-producing areas over a period of three years. Antonie van Leeuwenhoek 79, 345-352. doi: 10.1023/A:1012027718701

Viana, F., Belloch, C., Vallés, S., and Manzanares, P. (2011). Monitoring a mixed starter of Hanseniaspora vineae-Saccharomyces cerevisiae in natural must: impact on 2-phenylethyl acetate production. Int. J. Food Microbiol. 151, 235-240. doi: 10.1016/j.ijfoodmicro.2011.09.005

Viana, F., Gil, J. V., Vallés, S., and Manzanares, P. (2009). Increasing the levels of 2phenethyl acetate in wine through the use of a mixed culture of Hanseniaspora osmophila and Saccharomyces cerevisiae. Int. J. Food Microbiol. 135, 68-74. doi: 10.1016/j.ijfoodmicro.2009.07.025

Wang, C., Esteve-Zarzoso, B., Cocolin, L., Mas, A., and Rantsiou, K. (2015). Viable and culturable populations of Saccharomyces cerevisiae, Hanseniaspora uvarum and Starmerella bacillaris (synonym Candida zemplinina) during Barbera must fermentation. Food Res Int. 78, 195-200. doi: 10.1016/j.foodres.2015. 10.014

Wang, Q., Garrity, G. M., Tiedje, J. M., and Cole, J. R. (2007). Naïve Bayesian classifier for rapid assignment of rRNA sequences into the new bacterial taxonomy. Appl. Environ. Microb. 73, 5261-5267. doi: 10.1128/AEM.00 062-07

Conflict of Interest Statement: The authors declare that the research was conducted in the absence of any commercial or financial relationships that could be construed as a potential conflict of interest.

Copyright (C) 2016 Lleixà, Martín, Portillo, Carrau, Beltran and Mas. This is an open-access article distributed under the terms of the Creative Commons Attribution License (CC BY). The use, distribution or reproduction in other forums is permitted, provided the original author(s) or licensor are credited and that the original publication in this journal is cited, in accordance with accepted academic practice. No use, distribution or reproduction is permitted which does not comply with these terms. 